Pré-Publicações do Departamento de Matemática

Universidade de Coimbra

Preprint Number 13-02

\title{
DEFORMED LAGUERRE-HAHN ORTHOGONAL POLYNOMIALS ON THE REAL LINE
}

\author{
A. BRANQUINHO AND M.N. REBOCHO
}

\begin{abstract}
We study families of orthogonal polynomials on the real line whose Stieltjes functions satisfy a Riccati type differential equation with polynomial coefficients. We derive discrete dynamical systems, obtained as a result of deformations of the recurrence relation coefficients of the orthogonal polynomials related to the above referred Stieltjes functions.
\end{abstract}

KEYWORDS: Orthogonal polynomials, semi-classical class, matrix Sylvester differential equations, Lax pairs.

AMS Subject Classification (2000): 33C45, 39B42.

\section{Motivation}

The study of sequences of orthogonal polynomials on the real line whose Stieltjes function, $S$, satisfies a Riccati-type differential equation with polynomial coefficients

$$
A S^{\prime}=B S^{2}+C S+D,
$$

the so-called Laguerre-Hahn class, is of importance for many problems from applied mathematics and mathematical physics. For example, some of these families of orthogonal polynomials, the so-called co-recursive and generalized co-recursive polynomials, play an important role in the solution (of the Chapman-Kolmogorov equation) of birth and death processes with absorption or killing [13]. Other examples concern Hamiltonian operators: the study of perturbations on the corresponding physical system involve the analysis of orthogonal polynomials that satisfy a three term recurrence relation with perturbed initial conditions, thus leading to the analysis of Laguerre-Hahn families of orthogonal polynomials $[8,18]$.

In the present paper we derive discrete dynamical systems related to Laguerre-Hahn orthogonal polynomials. Such dynamical systems are obtained

Received January 18, 2013.

This work was partially supported by Centro de Matemática da Universidade de Coimbra (CMUC), funded by the European Regional Development Fund through the program COMPETE and by the Portuguese Government through the FCT - Fundação para a Ciência e a Tecnologia under the project PEst-C/MAT/UI0324/2011. M.N. Rebocho was also supported by grant ref. SFRH/BPD/45321/2008, from Fundação para a Ciência e Tecnologia (Portugal). 
as a result of deformations of the recurrence relation coefficients of the orthogonal polynomials under a $t$-dependence for the polynomials $A-D$ involved in the differential equation (1).

For the semi-classical case, that is, $B \equiv 0$ in (1), the connection between semi-classical orthogonal polynomials and integrable dynamical systems is well established (see [9]). There is a vast list of studies concerning deformation of the recurrence relation coefficients of the semi-classical orthogonal polynomials under evolution of the orthogonality weight, showing relations to random matrix theory, Lax pairs, Toda lattices, Painlevé equations, isomonodromic deformations, etc, and we refer the interested reader to $[3,4,6,10,11,12,15]$, as well as the references therein.

Our approach is based on the use of the equivalence between the differential equation (1) for the Stieltjes function, $S$, and the matrix Riccati equation

$$
A Y_{n}^{\prime}=\mathcal{B}_{n} Y_{n}-Y_{n} \mathcal{C}, \quad n \geq 0, \quad\left({ }^{\prime}=d / d x\right)
$$

for the corresponding $Y_{n}=\left[\begin{array}{cc}P_{n+1} & P_{n}^{(1)} \\ P_{n} & P_{n-1}^{(1)}\end{array}\right]$, with $\left\{P_{n}\right\}$ the sequence of monic orthogonal polynomials related to $S,\left\{P_{n}^{(1)}\right\}$ the sequence of associated polynomials of the first kind (cf. Theorem 1). Let us remark that the compatibility of the above differential system (2) and the matrix form of the recurrence relation yields a matrix Lax pair representation, leading to a discrete equation for the transfer matrices related to $\left\{P_{n}\right\}$ (cf. Eq. (10)). Such a discrete equation encloses nonlinear difference equations for the recurrence relation coefficients of the Laguerre-Hahn orthogonal polynomials, which can be viewed as comparable to the so-called Laguerre-Freud equations that hold for semi-classical families $[2,14]$. Furthermore, the evolution of the polynomials $A-D$ of (1), under dependence on a parameter $t$, leads to deformations of the recurrence relation coefficients that are described by differential equations in $t$. These will be analyzed in Theorems 3, 5 and 6 (see also [6], concerning related problems for orthogonal polynomials on the unit circle).

The paper is organized as follows. In section 2 we present the notations and basic results in the theory of orthogonal polynomials to be used in the forthcoming sections. In Section 3 we deduce the above mentioned discrete equations. In Section 4 we analyze the continuous differential equations in $t$ for the recurrence relation coefficients of the Laguerre-Hahn orthogonal polynomials. In Section 5 we present an example. 


\section{Preliminary results and notations}

Let $u$ be a linear form defined on the space of polynomials, $\mathbb{P}=\operatorname{span}\left\{x^{k}\right.$ : $\left.k \in \mathbb{N}_{0}\right\}$, and let $\left\{P_{n}(x)=x^{n}+\cdots\right\}$ be the sequence of monic orthogonal polynomials $(\mathrm{SMOP})$ related to $u$,

$$
\left\langle u, P_{n} P_{m}\right\rangle=h_{n} \delta_{n, m}, h_{n} \neq 0, \quad n, m \geq 0 .
$$

It is a basic result that a sequence of orthogonal polynomials related to $u$ exists if, and only if, the moments $u_{n}=\left\langle u, x^{n}\right\rangle, n \geq 0$ (where we take $u_{0}=1$ for matters of simplicity), satisfy $\operatorname{det} \Delta_{n} \neq 0, n \geq 0$, where $\Delta_{n}$ is the so-called Hankel matrix, $\Delta_{n}=\left[u_{i+j}\right]_{i, j=0}^{n}, n \geq 0$ (see [19]).

Furthermore, if $\operatorname{det} \Delta_{n}>0, n \geq 0$, then $u$ has an integral representation in terms of a positive Borel measure, $\mu$, supported on an infinite point set, $I$, of the real line,

$$
\left\langle u, x^{n}\right\rangle=\int_{I} x^{n} d \mu(x), \quad n \geq 0,
$$

and the orthogonality condition (3) becomes

$$
\int_{I} P_{n}(x) P_{m}(x) d \mu(x)=h_{n} \delta_{n, m}, \quad h_{n}>0, \quad n, m \geq 0 .
$$

If $\mu$ is an absolutely continuous measure supported on $I$, and $w$ denotes its Radon-Nikodym derivative with respect to the Lebesgue measure, i.e. $d \mu(x)=w(x) d x$, then we will also say that $\left\{P_{n}\right\}$ is orthogonal with respect to $w$.

It is well known that, a sequence of monic orthogonal polynomials, $\left\{P_{n}\right\}$, satisfies a three term recurrence relation (cf. [19])

$$
P_{n+1}(x)=\left(x-\beta_{n}\right) P_{n}(x)-\gamma_{n} P_{n-1}(x), \quad n=1,2, \ldots
$$

with $P_{0}(x)=1, P_{1}(x)=x-\beta_{0}$, and $\gamma_{n} \neq 0, n \geq 1, \gamma_{0}=1$.

The recurrence relation coefficients, $\gamma_{n}$ and $\beta_{n}$, are given by

$$
\gamma_{0}=u_{0}, \quad \gamma_{n+1}=\frac{\Delta_{n-1} \Delta_{n+1}}{\Delta_{n}^{2}}, \quad \beta_{n}=\frac{\left\langle u, x P_{n}^{2}\right\rangle}{\left\langle u, P_{n}^{2}\right\rangle}, n \geq 0, \Delta_{-1}=1 .
$$

Note that from (5) we get

$$
P_{n+1}(x)=x^{n+1}-\left(\xi_{n}+\beta_{0}\right) x^{n}+\left(\nu_{n}+\beta_{0} \xi_{n}-\gamma_{1}\right) x^{n-1}+\cdots,
$$

where

$$
\xi_{n}=\sum_{k=1}^{n} \beta_{k}, \quad \nu_{n}=\sum_{1 \leq i<j \leq n}^{n} \beta_{i} \beta_{j}-\sum_{k=2}^{n} \gamma_{k}, \quad n \geq 1
$$


We now define the associated polynomials of the first kind, by

$$
P_{n}^{(1)}(x)=\left\langle u_{s}, \frac{P_{n+1}(x)-P_{n+1}(s)}{x-s}\right\rangle, \quad n \geq 0,
$$

where $u_{s}$ denotes the action of $u$ on the variable $s$.

The sequence $\left\{P_{n}^{(1)}\right\}$ also satisfies a three term recurrence relation,

$$
P_{n}^{(1)}(x)=\left(x-\beta_{n}\right) P_{n-1}^{(1)}(x)-\gamma_{n} P_{n-2}^{(1)}(x), \quad n=1,2, \ldots
$$

with $P_{-1}^{(1)}(x)=0, P_{0}^{(1)}(x)=1$.

An important function in the theory of orthogonal polynomials is the Stieltjes function, also known as the generating function of the moments for $u$, given by

$$
S(x)=\sum_{n=0}^{+\infty} u_{n} x^{-n-1} .
$$

Note that if $u$ is defined by (4), then $S$ is given by

$$
S(x)=\int_{I} \frac{d \mu(s)}{x-s}, \quad x \in \mathbb{C} \backslash I .
$$

The sequence of functions of the second kind corresponding to $\left\{P_{n}\right\}$ is defined by

$$
q_{n+1}=P_{n+1} S-P_{n}^{(1)}, \quad n \geq 0, q_{0}=S,
$$

and in the positive-definite case,

$$
q_{n+1}(x)=\int_{I} \frac{P_{n+1}(s)}{x-s} d \mu(s), \quad n \geq 0, q_{0}=S .
$$

Definition 1. (see [16]) A Stieltjes function, $S$, is said to be Laguerre-Hahn if there exist polynomials $A, B, C, D$, with $A \neq 0$, such that it satisfies a Riccati differential equation

$$
A S^{\prime}=B S^{2}+C S+D .
$$

The corresponding sequence of orthogonal polynomials is called LaguerreHahn. If $B=0$, then $S$ is said to be Laguerre-Hahn affine or semi-classical.

The above polynomial $D$ can be written in terms of $A, B, C$ (see [16, eq. (7.7)]).

As it is well known, if $S$ is related to a positive-definite linear form $u$, in turn defined in terms of a weight $w$, then the semi-classical character of $S$, 
$A S^{\prime}=C S+D$, is equivalent to $A w^{\prime}=C w$, with $w$ (positive) satisfying the boundary conditions

$$
\left.x^{n} A(x) w(x)\right|_{a, b}=0, \quad n \geq 0,
$$

where $a, b$ (eventually $a$ or $b$ infinite) are linked with the roots of $A$ (see [19]). In such a case, $w$ is the weight function on the support $I=[a, b]$.

In the sequel we will use the following matrices:

$$
Y_{n}=\left[\begin{array}{cc}
P_{n+1} & P_{n}^{(1)} \\
P_{n} & P_{n-1}^{(1)}
\end{array}\right], \quad \widetilde{Y}_{n}=\left[\begin{array}{cc}
P_{n+1} & q_{n+1} / w \\
P_{n} & q_{n} / w
\end{array}\right], \quad Q_{n}=\left[\begin{array}{c}
q_{n+1} \\
q_{n}
\end{array}\right], \quad n \geq 0 .
$$

Lemma 1. Let $\left\{P_{n}\right\}$ be a SMOP and let $\beta_{n}, \gamma_{n}, n \geq 0$, be its recurrence relation coefficients. Let $\left\{Y_{n}\right\},\left\{\widetilde{Y}_{n}\right\},\left\{Q_{n}\right\}$ be the sequences defined in (7). Then, (a) $Y_{n}$ and $\widetilde{Y}_{n}$ satisfy the difference equation

$$
X_{n}=\mathcal{A}_{n} X_{n-1}, \quad \mathcal{A}_{n}=\left[\begin{array}{cc}
x-\beta_{n} & -\gamma_{n} \\
1 & 0
\end{array}\right], \quad n \geq 1
$$

with initial conditions $Y_{0}=\left[\begin{array}{cc}x-\beta_{0} & 1 \\ 1 & 0\end{array}\right], \quad \widetilde{Y}_{0}=\left[\begin{array}{cc}x-\beta_{0} & q_{1} / w \\ 1 & q_{0} / w\end{array}\right]$;

(b) $Q_{n}$ satisfies

$$
Q_{n}=\mathcal{A}_{n} Q_{n-1}, \quad n \geq 1,
$$

with $\mathcal{A}_{n}$ given in (8) and initial conditions $Q_{0}=\left[\begin{array}{c}\left(x-\beta_{0}\right) S-1 \\ S\end{array}\right]$.

The above matrix $\mathcal{A}_{n}$ is known in the literature as the transfer matrix of the SMOP.

Throughout the paper, $I$ denotes the $2 \times 2$ identity matrix. The $(i, j)$ entry of a matrix $X$ will be denoted by $X^{(i, j)}$. $f^{\prime}$ will denote the derivative of $f$ with respect to $x$ and $\dot{f}$ denotes the derivative of $f$ with respect to $t$.

\section{Differential systems for Laguerre-Hahn orthogonal polynomials and discrete Lax equations}

We begin by presenting a differential system for Laguerre-Hahn orthogonal polynomials. The result that follows was deduced in [5, Theorem 1], thus we omit here its proof. 
Theorem 1. Let $S$ be a Stieltjes function, let $\left\{Y_{n}\right\}$ and $\left\{Q_{n}\right\}$ be the corresponding sequences defined in (7), and let $\beta_{n}, \gamma_{n}$ be the corresponding recurrence relation coefficients. The following statements are equivalent:

(a) S satisfies

$$
A S^{\prime}=B S^{2}+C S+D, \quad A, B, C, D \in \mathbb{P}
$$

(b) $Y_{n}$ satisfies the matrix Sylvester equation

$$
A Y_{n}^{\prime}=\mathcal{B}_{n} Y_{n}-Y_{n} \mathcal{C}, \quad n \geq 0,
$$

where

$$
\mathcal{B}_{n}=\left[\begin{array}{cc}
l_{n} & \Theta_{n} \\
-\Theta_{n-1} / \gamma_{n} & l_{n-1}+\left(x-\beta_{n}\right) \Theta_{n-1} / \gamma_{n}
\end{array}\right], \mathcal{C}=\left[\begin{array}{cc}
C / 2 & -D \\
B & -C / 2
\end{array}\right]
$$

with $l_{n}, \Theta_{n}$ polynomials of uniformly bounded degrees, satisfying the initial conditions $A=\left(x-\beta_{0}\right)\left(l_{0}-C / 2\right)-B+\Theta_{0}, 0=\left(x-\beta_{0}\right) D+l_{0}+C / 2$, $\Theta_{-1}=D, l_{-1}=C / 2$;

(c) the transfer matrices, $\mathcal{A}_{n}=\left[\begin{array}{cc}x-\beta_{n} & -\gamma_{n} \\ 1 & 0\end{array}\right]$, satisfy

$$
A \mathcal{A}_{n}^{\prime}=\mathcal{B}_{n} \mathcal{A}_{n}-\mathcal{A}_{n} \mathcal{B}_{n-1}, \quad n \geq 1
$$

(d) $Q_{n}$ satisfies

$$
A Q_{n}^{\prime}=\left(\mathcal{B}_{n}+(B S+C / 2) I\right) Q_{n}, \quad n \geq 0 .
$$

Remark 1. Matrix Sylvester equations are particular cases of matrix Riccati equations (see $[1,17]$ ).

Remark 2. The discrete Lax equations (10) are obtained through the compatibility between the Lax pairs

$$
\left\{\begin{array}{l}
Y_{n}=\mathcal{A}_{n} Y_{n-1} \\
A Y_{n}^{\prime}=\mathcal{B}_{n} Y_{n}-Y_{n} \mathcal{C}, \quad n \geq 1 .
\end{array}\right.
$$

Note that (10) are equivalent to

$$
\left\{\begin{array}{l}
\left(x-\beta_{n}\right)\left(l_{n}-l_{n-1}\right)=A-\Theta_{n}+\frac{\gamma_{n}}{\gamma_{n-1}} \Theta_{n-2} \\
l_{n}-l_{n-2}=-\frac{\left(x-\beta_{n}\right)}{\gamma_{n}} \Theta_{n-1}+\frac{\left(x-\beta_{n-1}\right)}{\gamma_{n-1}} \Theta_{n-2}, \quad n \geq 1 .
\end{array}\right.
$$

As a consequence of the previous Theorem we have the result that follows (see [5, Corollary 1]). 
Corollary 1. The following relations hold:

$$
\operatorname{tr} \mathcal{B}_{n}=0, \quad n \geq 0 ;
$$

$\operatorname{det} \mathcal{B}_{n}=\operatorname{det} \mathcal{B}_{0}+A \sum_{k=1}^{n} \frac{\Theta_{k-1}}{\gamma_{k}}, n \geq 1$, with $\operatorname{det} \mathcal{B}_{0}=D(A+B)-(C / 2)^{2}$.

Theorem 2. Let $\left\{P_{n}\right\}$ be a SMOP with respect to a weight $w$, and let $\left\{q_{n}\right\}$ be the corresponding sequence of functions of the second kind. $w$ is semiclassical and satisfies $w^{\prime} / w=C / A$ if, and only if,

$$
\widetilde{Y}_{n}=\left[\begin{array}{cc}
P_{n+1} & q_{n+1} / w \\
P_{n} & q_{n} / w
\end{array}\right]: A \widetilde{Y}_{n}^{\prime}=\left(\mathcal{B}_{n}-\frac{C}{2} I\right) \widetilde{Y}_{n}, \quad n \geq 1,
$$

where $\mathcal{B}_{n}$ is the matrix associated with the equation $A S^{\prime}=C S+D$ for the Stieltjes function of $w$.

\section{Differential equations for deformed Laguerre-Hahn orthogonal polynomials}

Henceforth we consider Stieltjes functions satisfying differential equations $A S^{\prime}=B S^{2}+C S+D$, where $A, B, C, D$ are co-prime polynomials now depending on a parameter $t$ (we consider a general $t$-dependence). Notice that the corresponding orthogonal polynomials, as well as its recurrence relation coefficients, will also depend on $t$. For simplicity matters, henceforth we do not display the $t$-dependence on these quantities.

Theorem 3. Let $S$ be a Stieltjes function satisfying $A S^{\prime}=B S^{2}+C S+$ $D$, where $A, B, C, D$ are polynomials that depend on a parameter $t$, let the corresponding $\left\{Y_{n}\right\}$ satisfy the matrix Sylvester equations $A Y_{n}^{\prime}=\mathcal{B}_{n} Y_{n}-$ $Y_{n} \mathcal{C}, n \geq 1$, and let $\mathcal{L}$ be a nonsingular matrix such that $A \mathcal{L}^{\prime}=\mathcal{C} \mathcal{L}$. Then, the matrices $\mathcal{H}_{n}$ defined by

$$
\mathcal{H}_{n}=\left(\dot{Y}_{n}+Y_{n} \dot{\mathcal{L}} \mathcal{L}^{-1}\right) Y_{n}^{-1}, \quad n \geq 1
$$

satisfy

$$
\frac{\partial}{\partial t}\left(\frac{\mathcal{B}_{n}}{A}\right)=\mathcal{H}_{n}^{\prime}+\mathcal{H}_{n} \frac{\mathcal{B}_{n}}{A}-\frac{\mathcal{B}_{n}}{A} \mathcal{H}_{n}, \quad n \geq 1
$$

Furthermore, the transfer matrices of $Y_{n}$ satisfy

$$
\dot{\mathcal{A}}_{n}=\mathcal{H}_{n} \mathcal{A}_{n}-\mathcal{A}_{n} \mathcal{H}_{n-1}, \quad n \geq 2 .
$$


Proof: From (13), taking derivatives with respect to $x$, we obtain

$$
\begin{aligned}
\frac{\partial \dot{Y}_{n}}{\partial x}=\mathcal{H}_{n}^{\prime} Y_{n}+\mathcal{H}_{n}\left(\frac{\mathcal{B}_{n}}{A} Y_{n}\right. & \left.-Y_{n} \frac{\mathcal{C}}{A}\right)-\left(\frac{\mathcal{B}_{n}}{A} Y_{n}-Y_{n} \frac{\mathcal{C}}{A}\right) \dot{\mathcal{L}} \mathcal{L}^{-1} \\
& -Y_{n} \frac{\partial}{\partial t}\left(\frac{\mathcal{C}}{A}\right)-Y_{n} \frac{\mathcal{C}}{A} \dot{\mathcal{L}} \mathcal{L}^{-1}+Y_{n} \dot{\mathcal{L}} \mathcal{L}^{-1} \frac{\mathcal{C}}{A} .
\end{aligned}
$$

On the other hand, taking derivatives with respect to $t$ in $A Y_{n}^{\prime}=\mathcal{B}_{n} Y_{n}-Y_{n} \mathcal{C}$,

$$
\begin{aligned}
\frac{\partial Y_{n}^{\prime}}{\partial t}=\frac{\partial}{\partial t}\left(\frac{\mathcal{B}_{n}}{A}\right) Y_{n}+\frac{\mathcal{B}_{n}}{A}\left(\mathcal{H}_{n} Y_{n}\right. & \left.-Y_{n} \dot{\mathcal{L}} \mathcal{L}^{-1}\right) \\
& -\left(\mathcal{H}_{n} Y_{n}-Y_{n} \dot{\mathcal{L}} \mathcal{L}^{-1}\right) \frac{\mathcal{C}}{A}-Y_{n} \frac{\partial}{\partial t}\left(\frac{\mathcal{C}}{A}\right) .
\end{aligned}
$$

The compatibility of (16) and (17) yields

$$
\mathcal{H}_{n}^{\prime} Y_{n}+\mathcal{H}_{n} \frac{\mathcal{B}_{n}}{A} Y_{n}=\frac{\partial}{\partial t}\left(\frac{\mathcal{B}_{n}}{A}\right) Y_{n}+\frac{\mathcal{B}_{n}}{A} \mathcal{H}_{n} Y_{n} .
$$

Since $Y_{n}$ is nonsingular, we obtain (14).

To deduce (15) we take derivatives in $Y_{n}=\mathcal{A}_{n} Y_{n-1}$ (cf. (8)), and use (13) to $n-1, \dot{Y}_{n-1}=\mathcal{H}_{n-1} Y_{n-1}-Y_{n-1} \dot{\mathcal{L}} \mathcal{L}^{-1}$, thus obtaining

$$
\dot{\mathcal{A}}_{n} Y_{n-1}=\mathcal{H}_{n} \mathcal{A}_{n} Y_{n-1}-\mathcal{A}_{n} \mathcal{H}_{n-1} Y_{n-1} .
$$

Since $Y_{n}$ is nonsingular, we obtain (15).

Further properties of $\mathcal{H}_{n}$ follow.

Corollary 2. Let $\mathcal{H}_{n}$ be given by (13). Let $\gamma_{n}, \beta_{n}$ be the recurrence relation coefficients corresponding to $\left\{Y_{n}\right\}$. Then, for all $n \in \mathbb{N}$, tr $\mathcal{H}_{n}$ does not depend on $x$.

Furthermore, the following equations hold:

$$
\begin{gathered}
\operatorname{tr} \mathcal{H}_{n}=\sum_{k=1}^{n} \frac{\dot{\gamma}_{k}}{\gamma_{k}}+\operatorname{tr} \dot{\mathcal{L}} \mathcal{L}^{-1}, \quad n \geq 1 ; \\
\operatorname{det} \mathcal{H}_{n}=\operatorname{det} \mathcal{H}_{n-1}-\frac{\dot{\beta}_{n}}{\gamma_{n}} \mathcal{H}_{n-1}^{(1,2)}+\frac{\dot{\gamma}_{n}}{\gamma_{n}} \mathcal{H}_{n-1}^{(1,1)}, \quad n \geq 1 .
\end{gathered}
$$

Proof: From (14) we have

$$
\frac{\partial}{\partial t}\left(\frac{\mathcal{B}_{n}^{(1,1)}}{A}\right)=\left(\mathcal{H}_{n}^{(1,1)}\right)^{\prime}+\frac{1}{A}\left(\mathcal{B}_{n}^{(2,1)} \mathcal{H}_{n}^{(1,2)}-\mathcal{B}_{n}^{(1,2)} \mathcal{H}_{n}^{(2,1)}\right)
$$




$$
\frac{\partial}{\partial t}\left(\frac{\mathcal{B}_{n}^{(2,2)}}{A}\right)=\left(\mathcal{H}_{n}^{(2,2)}\right)^{\prime}+\frac{1}{A}\left(\mathcal{B}_{n}^{(1,2)} \mathcal{H}_{n}^{(2,1)}-\mathcal{B}_{n}^{(2,1)} \mathcal{H}_{n}^{(1,2)}\right)
$$

Summing (20) with (21) and using (11) we obtain

$$
\left(\operatorname{tr} \mathcal{H}_{n}\right)^{\prime}=0
$$

and we conclude that tr $\mathcal{H}_{n}$ does not depend on $x$.

The computation of the entries $(1,1)$ and $(2,2)$ of $\mathcal{H}_{n}$, from (13), yields

$$
\operatorname{tr} \mathcal{H}_{n}=\frac{\frac{\partial}{\partial t}\left(\operatorname{det} Y_{n}\right)}{\operatorname{det} Y_{n}}+\operatorname{tr} \dot{\mathcal{L}} \mathcal{L}^{-1}
$$

Since $\operatorname{det} Y_{n}=-\prod_{k=1}^{n} \gamma_{k}$, we obtain (18).

Eq. (19) follows from (15).

Remark 3. From (18) there follows that $\operatorname{tr} \dot{\mathcal{L}} \mathcal{L}^{-1}$ does not depend on $x$.

Note that it is of a great advantage to know information on the asymptotic expansion of the matrices $\mathcal{H}_{n}$ defined in (13), since the equations (14) and (15) enclose differential equations in $t$ for the recurrence relation coefficients of the corresponding Laguerre-Hahn orthogonal polynomials. It should be remarked that the matrix $\mathcal{H}_{n}$ depends on $\mathcal{L}$, which in turn is the solution of the differential system $A \mathcal{L}^{\prime}=\mathcal{C} \mathcal{L}, \mathcal{C}$ having polynomial entries.

Connection with semi-classical orthogonal polynomials: the use of Radon's Lemma. In what follows we establish a relation between $\mathcal{H}_{n}$ and a matrix (to be specified through the text) related to semi-classical families of orthogonal polynomials. We shall make use of a result known in the theory of matrix Riccati equations as Radon's Lemma [17]. In fact, for our purposes it is sufficient to show the following particular case of the Radon's Lemma (see [1] and [7, Theorem 5]).

Theorem 4. Let $A$ be a polynomial, let $\mathcal{B}_{n} / A, n \geq 1$, and $\mathcal{C} / A$ be matrices whose entries are integrable functions in a domain $G$ of the complex plane, and let $x_{0} \in G$. If the matrices $\mathcal{P}_{n}$ and $\mathcal{L}, \mathcal{L}$ nonsingular, satisfy

$$
\left\{\begin{array}{l}
A \mathcal{L}^{\prime}=\mathcal{C} \mathcal{L} \\
\mathcal{L}\left(x_{0}\right)=I
\end{array}\right.
$$


and

$$
\left\{\begin{array}{l}
A \mathcal{P}_{n}^{\prime}=\mathcal{B}_{n} \mathcal{P}_{n}, \quad n \geq 1, \\
\mathcal{P}_{n}\left(x_{0}\right)=X_{n}\left(x_{0}\right)
\end{array}\right.
$$

then the solution of $A X_{n}^{\prime}=\mathcal{B}_{n} X_{n}-X_{n} \mathcal{C}$, in $G$, is given by:

$$
X_{n}=\mathcal{P}_{n} \mathcal{L}^{-1}, \quad n \geq 1 \text {. }
$$

Recall that one has the Sylvester equation $A Y_{n}^{\prime}=\mathcal{B}_{n} Y_{n}-Y_{n} \mathcal{C}$, related to $A S^{\prime}=B S^{2}+C S+D(\mathrm{cf}$. Theorem 1). We start with some remarks on the solution of the corresponding differential system

$$
A \mathcal{P}_{n}^{\prime}=\mathcal{B}_{n} \mathcal{P}_{n}
$$

Hereafter we will consider $x_{0} \in \mathbb{C}$ and $\widetilde{C}$ a polynomial such that $\int_{x_{0}}^{x} \frac{\widetilde{C}(s)}{2 A(s)} d s$ is defined in suitable domains.

Lemma 2. Let $\mathcal{B}_{n}$ be matrices, and let $\widetilde{C}$ be a polynomial. A matrix $\widetilde{Y}_{n}$ satisfies

$$
A \widetilde{Y}_{n}^{\prime}=\left(\mathcal{B}_{n}-\widetilde{C} / 2 I\right) \widetilde{Y}_{n}
$$

if, and only if, $\mathcal{P}_{n}=e^{\int_{x_{0}}^{x} \frac{\widetilde{C}(s)}{2 A(s)} d s} \widetilde{Y}_{n}$ satisfies (22).

Taking into account the previous lemma, we will solve (22) by considering

$$
\mathcal{P}_{n}=e^{\int_{x_{0}}^{x} \frac{\widetilde{C}(s)}{2 A(s)} d s} \widetilde{Y}_{n}
$$

where $\widetilde{Y}_{n}$ satisfies (23). Furthermore, taking into account the Theorem 2, we will search for $\widetilde{Y}_{n}$ given by

$$
\widetilde{Y}_{n}=\left[\begin{array}{cc}
\widetilde{P}_{n+1} & \widetilde{q}_{n+1} / \widetilde{w} \\
\widetilde{P}_{n} & \widetilde{q}_{n} / \widetilde{w}
\end{array}\right]
$$

with $\left\{\widetilde{P}_{n}\right\}$ a SMOP with respect to a weight function $\widetilde{w}$ and $\left\{\widetilde{q}_{n}\right\}$ the corresponding sequence of functions of the second kind. Note that (23) implies

$$
\left(\operatorname{det} \widetilde{Y}_{n}\right)^{\prime}=\frac{\operatorname{tr}\left(\mathcal{B}_{n}-\widetilde{C} / 2 I\right)}{A} \operatorname{det} \widetilde{Y}_{n},
$$

which combined with $\operatorname{det} \widetilde{Y}_{n}=\left(\prod_{k=1}^{n} \widetilde{\gamma}_{k}\right) / w$ yields

$$
\widetilde{w}^{\prime} / \widetilde{w}=\widetilde{C} / A
$$

thus

$$
\widetilde{w}=e^{\int \widetilde{C} / A} .
$$


Theorem 5. Let $S$ be a Stieltjes function satisfying $A S^{\prime}=B S^{2}+C S+D$, where $A-D$ are co-prime polynomials that depend on a parameter $t$. Let $A Y_{n}^{\prime}=\mathcal{B}_{n} Y_{n}-Y_{n} \mathcal{C}$ be the corresponding matrix Sylvester equations, and consider the systems $A \mathcal{P}_{n}^{\prime}=\mathcal{B}_{n} \mathcal{P}_{n}, A \mathcal{L}^{\prime}=\mathcal{C} \mathcal{L}$. Let $G$ be a domain in the complex plane such that the entries of the matrices $\mathcal{B}_{n} / A$ and $\mathcal{C} / A$ are integrable in $G$, and let $x_{0} \in G$. Let the following assumption hold:

$$
\exists \widetilde{C} \in \mathbb{P}, \exists n_{0} \geq 1: \mathcal{P}_{n}=e^{\int_{x_{0}}^{x} \frac{\tilde{C}(s)}{2 A(s)} d s} \widetilde{Y}_{n-n_{0}}, \quad n \geq n_{0}+1,
$$

with $\widetilde{Y}_{n}=\left[\begin{array}{cc}\widetilde{P}_{n+1} & \widetilde{q}_{n+1} / \widetilde{w} \\ \widetilde{P}_{n} & \widetilde{q}_{n} / \widetilde{w}\end{array}\right]$, where $\left\{\widetilde{P}_{n}\right\}$ is the SMOP with respect to $\widetilde{w}=$ $e^{\int_{x_{0}}^{x} \frac{\tilde{C}(s)}{A(s)} d s}$. Then, for $x \in G$,

$$
Y_{n}=e^{\int_{x_{0}}^{x} \frac{\widetilde{C}(s)}{A(s)} d s}\left[\begin{array}{cc}
\widetilde{P}_{n-n_{0}+1} & \widetilde{q}_{n-n_{0}+1} / \widetilde{w} \\
\widetilde{P}_{n-n_{0}} & \widetilde{q}_{n-n_{0}} / \widetilde{w}
\end{array}\right] \mathcal{L}^{-1}, \quad n \geq n_{0}+1 .
$$

Furthermore, the matrices $\mathcal{H}_{n}$ defined in (13) are given by

$$
\mathcal{H}_{n}=\frac{1}{2}\left(\frac{\partial \widetilde{w}}{\partial t}\right) / \widetilde{w} I+\widetilde{\mathcal{H}}_{n-n_{0}}
$$

with the $\widetilde{\mathcal{H}}_{n}$ 's defined by

$$
\widetilde{\mathcal{H}}_{n}=\frac{\partial \widetilde{Y}_{n}}{\partial t} \widetilde{Y}_{n}^{-1}
$$

Proof: The representation (25) follows from

$$
Y_{n}=\mathcal{P}_{n} \mathcal{L}^{-1}, \quad n \geq 1,
$$

stated in Theorem 4, combined with the assumption (24). Taking derivatives with respect to $t$ in $(27)$ we get

$$
\dot{Y}_{n}=\mathcal{F}_{n} Y_{n}-Y_{n} \dot{\mathcal{L}} \mathcal{L}^{-1}, \quad \mathcal{F}_{n}=\dot{\mathcal{P}}_{n} \mathcal{P}_{n}^{-1},
$$

and the comparison between (28) and (13) yields

$$
\mathcal{H}_{n}=\dot{\mathcal{P}}_{n} \mathcal{P}_{n}^{-1}
$$

Using the assumption (24) written as $\mathcal{P}_{n}=\sqrt{\widetilde{w}} \widetilde{Y}_{n-n_{0}}$, (29) yields (26). 
Lemma 3. Let $\left\{\widetilde{P}_{n}\right\}$ be the SMOP related to the semi-classical weight $\widetilde{w}$ satisfying $\widetilde{w}^{\prime} / \widetilde{w}=\widetilde{C} / A$, with the conditions:

(i) $A(x)=\prod_{k=1}^{m}\left(x-x_{k}\right), x_{i} \neq x_{j}, i \neq j$,

(ii) the residues $\epsilon_{k}=\widetilde{C}\left(x_{k}\right) / A^{\prime}\left(x_{k}\right)$ are not integers, $k=1, \ldots, m$.

Let the following differential system hold:

$$
A \widetilde{Y}_{n}^{\prime}=\left(\widetilde{\mathcal{B}}_{n}-\widetilde{C} / 2\right) \widetilde{Y}_{n}, \quad \widetilde{Y}_{n}=\left[\begin{array}{cc}
\widetilde{P}_{n+1} & \widetilde{q}_{n+1} / \widetilde{w} \\
\widetilde{P}_{n} & \widetilde{q}_{n} / \widetilde{w}
\end{array}\right], \quad n \geq 1 .
$$

The matrices $\widetilde{\mathcal{H}}_{n}=\frac{\partial \widetilde{Y}_{n}}{\partial t} \widetilde{Y}_{n}^{-1}$ are given by

$$
\widetilde{\mathcal{H}}_{n}(x)=\widetilde{\mathcal{H}}_{\infty, n}-\sum_{k=1}^{m} \frac{\Re e s\left(\left(\widetilde{\mathcal{B}}_{n}-\widetilde{C} / 2\right) / A ; x=x_{k}\right)}{x-x_{k}} \dot{x}_{k}, \quad n \geq 1
$$

with $\widetilde{\mathcal{H}}_{\infty, n}=\left[\begin{array}{cc}0 & 0 \\ 0 & \frac{d \tilde{h}_{n}}{d t}\end{array}\right]$ and $\Re$ es $\left(\left(\widetilde{\mathcal{B}}_{n}-\widetilde{C} / 2\right) / A ; x=x_{k}\right)$ denoting the residue matrix of $\left(\widetilde{\mathcal{B}}_{n}-\widetilde{C} / 2\right) / A$ at $x_{k}$.

Proof: The detailed proof of (30) proceeds in similarity with [15, Section 3]. Note that we use $\widetilde{q}_{n}(x)=\tilde{h}_{n} x^{-n-1}+\mathcal{O}\left(x^{-n-2}\right)$, with $\tilde{h}_{n}$ given in the corresponding orthogonality relation (3), and also note that the $\widetilde{P}_{n}$ 's are monic.

\section{Examples}

Lemma 4. Let $\left\{P_{n}\right\}$ be the $S M O P$ related to $A S^{\prime}=B S^{2}+C S+D, A(x)=$ $a_{3} x^{3}+a_{2} x^{2}+a_{1} x+a_{0}, B(x)=b_{3} x^{3}+b_{2} x^{2}+b_{1} x+b_{0}, C(x)=c_{2} x^{2}+c_{1} x+$ $c_{0}, D(x)=d_{1} x+d_{0}$. Let $\beta_{n}, \gamma_{n}, n \geq 0$, be the recurrence relation coefficients of $\left\{P_{n}\right\}$. Then,

$$
d_{1}=-a_{3}-b_{3}-c_{2}, \quad d_{0}=-a_{2}-b_{2}-c_{1}+\beta_{0}\left(-2 a_{3}-2 b_{3}-c_{2}\right),
$$

and the matrices $\mathcal{B}_{n}=\left[\begin{array}{cc}l_{n} & \Theta_{n} \\ -\Theta_{n-1} / \gamma_{n} & -l_{n}\end{array}\right]$ involved in the corresponding Sylvester equations (9) are such that $l_{n}(x)=\ell_{n, 2} x^{2}+\ell_{n, 1} x+\ell_{n, 0}, \Theta_{n}(x)=$ 

$\theta_{n, 1} x+\theta_{n, 0}$, where, for all $n \geq 1$

$$
\begin{aligned}
& \ell_{n, 2}= c_{2} / 2+(n+1) a_{3}+b_{3}, \\
& \ell_{n, 1}= c_{1} / 2+\xi_{n} a_{3}+(n+1) a_{2}+\beta_{0}\left(a_{3}+b_{3}\right)+b_{2}, \\
& \ell_{n, 0}= c_{0} / 2-2 \nu_{n} a_{3}+(n+1) a_{1}+\gamma_{1} b_{3}+\xi_{n} a_{2}+\xi_{n}^{2} a_{3}+\beta_{0}^{2}\left(a_{3}+b_{3}\right) \\
& \quad+\beta_{0} b_{2}+b_{1}+\gamma_{n+1}\left[c_{2}+(2 n+3) a_{3}+2 b_{3}\right], \\
& \theta_{n, 1}=-\gamma_{n+1}\left[c_{2}+(2 n+3) a_{3}+2 b_{3}\right], \\
& \theta_{n, 0}=-\gamma_{n+1}\left[c_{1}+(2 n+3) a_{2}+a_{3}\left(\xi_{n}+\xi_{n+1}\right)+2 \beta_{0}\left(a_{3}+b_{3}\right)+2 b_{2}\right] \\
& \quad+\beta_{n+1} \theta_{n, 1},
\end{aligned}
$$

where

$$
\xi_{n}=\sum_{k=1}^{n} \beta_{k}, \quad \nu_{n}=\sum_{1 \leq i<j \leq n}^{n} \beta_{i} \beta_{j}-\sum_{k=2}^{n} \gamma_{k}, \quad n \geq 1 .
$$

Furthermore, the following holds:

$$
\gamma_{1}=\frac{-a_{1}-b_{1}-c_{0}-\beta_{0}\left[2 b_{2}+c_{1}+a_{2}+\beta_{0}\left(3 a_{3}+3 b_{3}+c_{2}\right)\right]}{a_{3}+2 b_{3}+c_{2}} .
$$

Proof: Use the representation (6) as well as

$$
P_{n}^{(1)}(x)=x^{n}-\xi_{n} x^{n-1}+\nu_{n} x^{n-2}+\cdots
$$

and compare the coefficients of the corresponding monomials.

For later purposes we show some results concerning the modified Laguerre polynomials, orthogonal with respect to the semi-classical weight

$$
\widetilde{w}(x)=(1-\zeta H(x-t))|x-t|^{\alpha} x^{\mu} e^{-x}
$$

supported on $\mathbb{R}^{+}, \zeta<1$, where $H$ denotes the Heaviside function, $H(y)=1$ for $y>0, H(y)=0$ otherwise.

Lemma 5. Let $\left\{\widetilde{P}_{n}\right\}$ be the SMOP related to the semi-classical weight (36). Let $\widetilde{\beta}_{n}, \widetilde{\gamma}_{n}, n \geq 0$, denote the recurrence relation coefficients of $\left\{\widetilde{P}_{n}\right\}$, let $\left\{\widetilde{q}_{n}\right\}$ be the corresponding sequence of functions of the second kind. The following statements hold:

(a) the Stieltjes function, $\widetilde{S}$, related to $\left\{\widetilde{P}_{n}\right\}$ satisfies

$$
A \widetilde{S^{\prime}}=\widetilde{C} \widetilde{S}+\widetilde{D}
$$


$A(x)=x^{2}-t x, \widetilde{C}(x)=-x^{2}+(\alpha+\mu+t) x-\mu t, \widetilde{D}(x)=x+\left(\widetilde{\beta}_{0}-1-\alpha-\mu-t\right) ;$ (b) $\widetilde{Y}_{n}=\left[\begin{array}{cc}\widetilde{P}_{n+1} & \widetilde{q}_{n+1} / \widetilde{w} \\ \widetilde{P}_{n} & \widetilde{q}_{n} / \widetilde{w}\end{array}\right]$ satisfies $A \widetilde{Y}_{n}^{\prime}=\left(\widetilde{\mathcal{B}}_{n}-\frac{\widetilde{C}}{2} I\right) \widetilde{Y}_{n}, n \geq 1$, where the entries of $\widetilde{\mathcal{B}}_{n}$ are defined by

$$
\begin{aligned}
& \widetilde{l}_{n}(x)=-\frac{x^{2}}{2}+\left(\frac{\alpha+\mu+t}{2}+n+1\right) x-\frac{\mu t}{2}-(n+1) t+\widetilde{\xi}_{n}-\widetilde{\gamma}_{n+1}, \\
& \widetilde{\Theta}_{n}(x)=\widetilde{\gamma}_{n+1} x-\widetilde{\gamma}_{n+1}\left(\alpha+\mu+t+2 n+3-\widetilde{\beta}_{n+1}\right),
\end{aligned}
$$

where $\widetilde{\xi}_{n}=\sum_{k=1}^{n} \widetilde{\beta}_{k}$.

Proof: Note that the logarithmic derivative of $\widetilde{w}$ given by (36) coincides with the logarithmic derivative of $(x-t)^{\alpha} x^{\mu} e^{-x}$ almost everywhere, and so it is independent of $\zeta$, that is,

$$
\widetilde{w}^{\prime} / \widetilde{w}=\left(-x^{2}+(\alpha+\mu+t) x-\mu t\right) /\left(x^{2}-t x\right) .
$$

Therefore, the equation (37) for the corresponding Stieltjes function follows. The differential system for $\widetilde{Y}_{n}$ follows from the Theorem 2 (cf. (12)), where the entries of $\widetilde{\mathcal{B}}_{n}$ are obtained using (31)-(35).

Lemma 6. Let $\left\{P_{n}\right\}$ be the SMOP related to the Stieltjes function $S$ satisfying $A S^{\prime}=B S^{2}+C S+D$, where

$$
\begin{aligned}
& A(x)=x^{2}-t x \\
& B(x)=-x^{3}+\left(\alpha+\mu+t+\beta_{0}-1\right) x^{2}+\beta_{0} \mu t \\
& \quad+\left(-\mu t-\beta_{0}(\alpha+\mu+t)+\gamma_{1}+t\right) x+\gamma_{1}\left(\beta_{0}-1-\alpha-\mu-t\right), \\
& C(x)=x^{2}-(\alpha+\mu+t) x+\mu t \\
& D(x)=0
\end{aligned}
$$

Let $\beta_{n}, \gamma_{n}, n \geq 0$, denote the recurrence relation coefficients of $\left\{P_{n}\right\}$, with $\beta_{0}=0, \gamma_{1}=\left(t-\beta_{1}\right) /(t-1)$. Then, $Y_{n}=\left[\begin{array}{cc}P_{n+1} & P_{n}^{(1)} \\ P_{n} & P_{n-1}^{(1)}\end{array}\right]$ satisfies the matrix Sylvester equation $Y_{n}^{\prime}=\mathcal{B}_{n} Y_{n}-\mathcal{C} Y_{n}, n \geq 1$, where the entries of $\mathcal{B}_{n}$ are defined by

$$
\begin{aligned}
& l_{n}(x)=-\frac{x^{2}}{2}+\left(\frac{\alpha+\mu+t}{2}+n\right) x-\frac{\mu t}{2}-n t+\xi_{n}-\gamma_{n+1}-\beta_{1}, \\
& \Theta_{n}(x)=\gamma_{n+1} x-\gamma_{n+1}\left(\alpha+\mu+t+2 n+1-\beta_{n+1}\right) .
\end{aligned}
$$


Proof: Use equations (31)-(35).

Lemma 7. Let $S$ be a Stieltjes function satisfying $A S^{\prime}=B S^{2}+C S+D$ with $D \equiv 0$, where $A-D$ are co-prime polynomials that depend on a parameter $t$. Let $x_{0}$ belong to $\{x \in \mathbb{C}: A(x) \neq 0\}$. Let the matrix $\mathcal{L}$ satisfy

$$
\left\{\begin{array}{l}
A \mathcal{L}^{\prime}=\mathcal{C} \mathcal{L}, \quad \mathcal{C}=\left[\begin{array}{cc}
C / 2 & 0 \\
B & -C / 2
\end{array}\right] \\
\mathcal{L}\left(x_{0}, \cdot\right)=I
\end{array}\right.
$$

There holds

$$
\dot{\mathcal{L}} \mathcal{L}^{-1}=\frac{\mathcal{E}}{A}, \quad \mathcal{E}=\left[\begin{array}{cc}
E / 2 & 0 \\
\frac{A}{W} \int_{x_{0}}^{x} \frac{\partial}{\partial t}\left(\frac{B}{A} W\right) d s & -E / 2
\end{array}\right],
$$

where $W=e^{\int_{x_{0}}^{x} \frac{C(s)}{A(s)} d s}$, and $E=A \frac{\dot{W}}{W}$.

Proof: The solution of (42) is

$$
\mathcal{L}=\left[\begin{array}{cc}
W^{1 / 2} & 0 \\
\left(\int_{x_{0}}^{x} \frac{B(s)}{A(s)} W(s) d s\right) W^{-1 / 2} & W^{-1 / 2}
\end{array}\right] .
$$

Therefore, (43) follows.

Theorem 6. Let $\left\{P_{n}\right\}$ be the SMOP related to the Stieltjes function $S$ satisfying $A S^{\prime}=B S^{2}+C S+D$, with $A-D$ given by (38)-(41). The transfer matrix of $P_{n}, \mathcal{A}_{n}=\left[\begin{array}{cc}x-\beta_{n} & -\gamma_{n} \\ 1 & 0\end{array}\right]$, satisfies the equation

$$
\dot{\mathcal{A}}_{n}=\mathcal{H}_{n} \mathcal{A}_{n}-\mathcal{A}_{n} \mathcal{H}_{n-1}, \quad n \geq 2,
$$

with

$$
\begin{aligned}
& \mathcal{H}_{n}=\left[\begin{array}{cc}
0 & 0 \\
0 & \frac{d \tilde{h}_{n-1}}{d t}
\end{array}\right] \\
& -\frac{1}{x-t}\left[\begin{array}{cc}
\alpha / 2+\left(\widetilde{\xi}_{n-1}-\widetilde{\gamma}_{n}\right) / t & -\widetilde{\gamma}_{n}\left(\alpha+\mu+2 n+1-\widetilde{\beta}_{n}\right) / t \\
\left(\alpha+\mu+2 n-1-\widetilde{\beta}_{n-1}\right) / t & -\alpha / 2-\left(\widetilde{\xi}_{n-1}-\widetilde{\gamma}_{n}\right) / t
\end{array}\right],
\end{aligned}
$$

where $\widetilde{\beta}_{n}, \widetilde{\gamma}_{n}$ are the recurrence relation coefficients of the SMOP $\left\{\widetilde{P}_{n}\right\}$ related to the weight $\widetilde{w}$ defined by $\widetilde{w}^{\prime} / \widetilde{w}=-C / A$. 
Thus, the recurrence relation coefficients of $\left\{P_{n}\right\}$ satisfy, for all $n \geq 2$,

$$
\begin{aligned}
& \frac{\dot{\gamma}_{n}}{\gamma_{n}}=\frac{2+\widetilde{\beta}_{n-2}-\widetilde{\beta}_{n-1}}{t}, \\
& \dot{\beta}_{n}=\frac{\widetilde{\gamma}_{n-1}-\widetilde{\gamma}_{n}+\widetilde{\beta}_{n-1}}{t} .
\end{aligned}
$$

Proof: Note that the assumption (24) of Theorem 5 holds, with $\widetilde{C}=-C$ and $n_{0}=1$, thus $\widetilde{w}$ given by (36). Therefore, the formula (26) for $\mathcal{H}_{n}$, combined with the asymptotic expansion (30), is

$$
\mathcal{H}_{n}=\frac{1}{2}\left(\frac{\partial \widetilde{w}}{\partial t}\right) / \widetilde{w} I+\left[\begin{array}{cc}
0 & 0 \\
0 & \frac{d \tilde{h}_{n-1}}{d t}
\end{array}\right]-\frac{\Re e s\left(\left(\widetilde{\mathcal{B}}_{n}-\widetilde{C} / 2\right) / A ; x=t\right)}{x-t},
$$

since there is only one zero of $A$ depending on $t$. Thus, (45) follows.

Let us use (45) in (44) and compute the limit at $x=\infty$. The position $(2,2)$ gives us $\gamma_{n}=\widetilde{\gamma}_{n-1}$. The use of the preceding equality in the resulting equations of positions $(1,2)$ and $(2,1)$ yield $(46)$, and the resulting equations from position $(1,1)$ yield $(47)$.

\section{References}

[1] H. Abou-Kandil, G. Freiling, V. Ionescu, and G. Jank, Matrix Riccati Equations in Control and Systems Theory, Birkhäuser, 2003.

[2] S. Belmehdi, A. Ronveaux, Laguerre-Freud's equations for the recurrence coefficients of semiclassical orthogonal polynomials, J. Approx. Theory, 76 (3)(1994), 351-368.

[3] P. Bleher and A. Its, Semiclassical asymptotics of orthogonal plynomials, Riemann-Hilbert problem, and universality in the matrix model, Annals of Mathematics, 150 (1999), 185-266.

[4] A.I. Aptekarev, A. Branquinho, and F. Marcellán, Toda-Type Differential Equations for the Recurrence Coefficients of Orthogonal Polynomials and Freud Transformation, J. Comput. Appl. Math. 78 (1997), 139-160.

[5] A. Branquinho, A. Paiva, and M.N. Rebocho, Sylvester equations for Laguerre-Hahn orthogonal polynomials on the real line, submitted (available at http://www.mat.uc.pt/ preprints/ps/p1224.pdf)

[6] A. Branquinho and M.N. Rebocho, Difference and differential equations for deformed LaguerreHahn orthogonal polynomials on the unit circle, J. Phys. A: Math. Theor. 44 (2011) 465204.

[7] A. Branquinho and M.N. Rebocho, Matrix Sylvester equations in the theory of orthogonal polynomials on the unit circle, Bull. Belg. Math. Soc. Simon Stevin 17 (2010) 355-376.

[8] T. S. Chihara, On co-recursive orthogonal polynomials, Proc. Amer. Math. Soc. 8 (1957) 899905.

[9] D.V. Chudnovsky, Riemann monodromy problem, isomonodromy deformation equations and completely integrable systems, pp. 385-447 in Bifurcation Phenomena in Mathematical Physics and Related Topics, Proceedings Cargése, 1979 (C. Bardos and D. Bessis, editors), NATO ASI series C, 54, D. Reidel, Dordrecht, 1980. 
[10] P.J. Forrester and C.M. Ormerod, Differential equations for deformed Laguerre polynomials, J. Approx. Theory 162 (4) (2010), 653-677.

[11] P.J. Forrester and N.S. Witte, Bi-orthogonal Polynomials on the Unit Circle, Regular SemiClassical Weights and Integrable Systems, Constr. Approx. 24 (2) (2006), 201-237.

[12] P.J. Forrester and N.S. Witte, Discrete Painlevé equations for a class of PVI $\tau$-functions given as $U(N)$ averages (2004), Nonlinearity 18 (2005), 2061-2088.

[13] M.E. Ismail, J. Letessier, D. Masson, and G. Valent, Birth and death processes and orthogonal polynomials, in Orthogonal Polynomials: Theory and Practice, P. Nevai, ed., vol. 294, NATO ASI series C, Kluwer, Dordrecht, Boston, London, 1990, pp. 229-255.

[14] A.P. Magnus, On Freud's equations for exponential weights, J. Approx. Theory 46 (1986) 65-99.

[15] A.P. Magnus, Painlevé-type differential equations for the recurrence coefficients of semiclassical orthogonal polynomials, J. Comput. Appl. Math. 57 (1995), 215-237.

[16] P. Maroni, Une théorie algébrique des polynômes orthogonaux. Application aux polynômes orthogonaux semi-classiques. In Orthogonal polynomials and their applications (Erice 1990), 95-130, IMACS Ann. Comput. Appl. Math. 9, Baltzer, Basel, 1991.

[17] J. Radon, Zum Problem von Lagrange, Hamburger Math. Einzelschr., 6, 1928.

[18] H. A. Slimm, On co-recursive orthogonal polynomials and their application to potential scattering, J. Math. Anal. Appl. 136 (1988) 1-19.

[19] G. Szegö, Orthogonal Polynomials, Amer. Math. Soc. Colloq. Publ., vol. 23, Amer. Math. Soc. Providence Rhode Island, 1975 (fourth edition).

A. Branquinho

CMUC and Department of Mathematics, University of Coimbra, Apartado 3008, EC Santa

Cruz, 3001-501 COIMBRA, Portugal.

E-mail address: ajplb@mat.uc.pt

M.N. REBOCHO

CMUC and Department of Mathematics, University of Beira Interior, 6201-001 Covilhã, PORTUGAL.

E-mail address: mneves@ubi.pt 